\title{
Analysis of the sulfur-regulated control of the cystathionine $\gamma$-lyase gene of Neurospora crassa
}

\author{
Brad S Reveal and John V Paietta
}

\begin{abstract}
Background: Cystathionine $\gamma$-lyase plays a key role in the transsulfuration pathway through its primary reaction of catalyzing the formation of cysteine from cystathionine. The Neurospora crassa cystathionine $\gamma$-lyase gene (cys-16 $\left.6^{+}\right)$ is of particular interest in dissecting the regulation and dynamics of transsulfuration. The aim of this study was to determine the regulatory connection of cys- $16^{+}$to the Neurospora sulfur regulatory network. In addition, the cys-16 promoter was characterized with the goal of developing a strongly expressed and regulatable gene expression tool.

Findings: The cystathionine $\gamma$-lyase cys- $16^{+}$gene was cloned and characterized. The gene, which contains no introns, encodes a protein of 417 amino acids with conserved pyridoxal 5'-phosphate binding site and substratecofactor binding pocket. Northern blot analysis using wild type cells showed that cys-16 $6^{+}$transcript levels increased under sulfur limiting (derepressing) conditions and were present only at a low level under sulfur sufficient (repressing) conditions. In contrast, cys-16 $6^{+}$transcript levels in a $\Delta$ cys-3 regulatory mutant were present at a low level under either derepressing or repressing conditions. Gel mobility shift analysis demonstrated the presence of four CYS3 transcriptional activator binding sites on the cys- $16^{+}$promoter, which were close matches to the CYS3 consensus binding sequence.

Conclusions: In this work, we confirm the control of cystathionine $y$-lyase gene expression by the CYS3 transcriptional activator through the loss of cys- $16^{+}$expression in a $\Delta$ cys-3 mutant and through the in vitro binding of CYS3 to the cys- $16^{+}$promoter at four sites. The highly regulated cys- $16^{+}$promoter should be a useful tool for gene expression studies in Neurospora
\end{abstract}

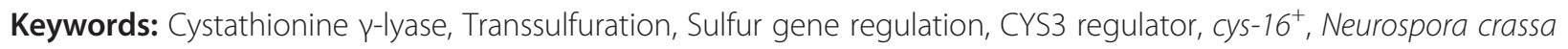

\section{Findings}

\section{Background}

Cystathionine $\gamma$-lyase (E.C. 4.4.1.1; also known as $\gamma$ cystathionase) catalyzes the conversion of cystathionine to cysteine and $\alpha$-ketobutryrate. Cystathionine $\gamma$-lyase, therefore, plays a key role in the transsulfuration reactions involved in the interconversion of homocysteine and cysteine routing through the intermediate cystathionine. Secondary reactions catalyzed include elimination reactions of homoserine, cystine, and cysteine; which in the latter case yield hydrogen sulfide $\left(\mathrm{H}_{2} \mathrm{~S}\right)$. The proposed role of $\mathrm{H}_{2} \mathrm{~S}$ as a physiologic signaling molecule has led to a number studies with the mammalian cystathionine $\gamma$-lyase [1,2], including gene knock-out constructs in mice [3]. Interestingly, the reverse

\footnotetext{
* Correspondence: john.paietta@wright.edu

Department of Biochemistry and Molecular Biology, Wright State University, Dayton, $\mathrm{OH}$ 45435, USA
}

transsulfuration pathway using cystathionine $\beta$-synthase and cystathionine $\gamma$-lyase, appears restricted to fungal and mammalian systems among eukaryotes $[4,5]$. Thus, the fungi offer an additional model system for cystathionine $\gamma$-lyase studies.

The properties and expression of cystathionine $\gamma$-lyase in fungi have been examined in several species. In Neurospora crassa the enzyme has been purified [6] and enzyme assays have shown up to a 30x increase in activity upon sulfur starvation $[7,8]$ as well as low activity under sulfur sufficient conditions [8]. In Aspergillus nidulans, the cystathionine $\gamma$-lyase gene (designated $m e c B$ ) has been cloned; $m е c B$ transcript levels show derepression under low sulfate levels; but showed increased transcription (with proportional enzyme activity increases) following growth with methionine supplementation [9]. In contrast, the Acremonium chrysogenum cystathionine $\gamma$ lyase gene does not appear to be regulated by the level of

\section{Biomed Central}


methionine [10]. In N. crassa, unlike these other fungi, the sulfur level provided for growth appears to have a straightforward effect on either derepressing or repressing cystathionine $\gamma$-lyase enzyme activity level [8].

The $N$. crassa sulfur regulatory system is composed of a genetically defined set of trans-acting regulatory genes and a set of structural genes encoding enzymes used in the uptake and assimilation of a variety of sulfur compounds [11-13]. Basically, when N. crassa is cultured under conditions of sulfur limitation (i.e., derepressing conditions) then an entire set of sulfur-related enzymes is coordinately expressed. The available data [8], therefore, suggests that $N$. crassa cystathionine $\gamma$-lyase is likely under control of this control system; along with other confirmed sulfur-regulated genes encoding arylsulfatase, sulfate permeases I and II, choline sulfatase, and others. The key regulator in the control system is the CYS3 bZIP transcriptional activator which is essential for sulfur structural gene expression [11]. The consensus binding sequence for CYS3 has been determined by in vitro binding-site selection studies [14].

Besides the cystathionine $\gamma$-lyase enzymatic assays done under sulfur-limiting and sulfur -sufficient conditions mentioned above, there is additional data available from microarray studies that have examined gene expression during Neurospora crassa development that include $c y s-16^{+}$transcript levels. A major increase in cys$16^{+}$transcript level was observed during the initial phase of the conidial germination process [15]. Starting with dormant conidia, there was an approximately 2.5 -fold increase in cys-16 transcript level at 1 hour into the germination process [15]. The cys- $16^{+}$transcript level then slowly declined, from the peak level seen at 1 hour, over an incubation time of 16 hours to end up slightly below the starting level (i.e., that seen in dormant conidia). The elevation in cys- $16^{+}$transcript (and presumably cystathionine $\gamma$-lyase activity) seems likely to represent an accession of the available cystathionine pool in order to supply the cellular demand for cysteine as germination commences and active growth begins. Consistent with this idea, additional microarray studies demonstrated a 3.6-fold higher cys- $16^{+}$transcript level in the active growth periphery (i.e., hyphal tips) as compared to a colonies interior region (i.e., 12-15 hours old) [16]. A final note regarding available microarray data is that a 2-fold increase in cys-16 $16^{+}$transcript level was observed post-exposure to phytosphingosine (which induces programmed cell death and notable increases in transcript level for many metabolism-related genes) [17]. The microarray data provides a useful developmental context for future studies of cys- $16^{+}$.

In this report, we present the cloning, transcript and promoter analysis of the $N$. crassa cystathionine $\gamma$-lyase $\left(\right.$ cys- $\left.16^{+}\right)$gene. The data extend the role of the CYS3- directed regulatory system to include the transsulfuration pathway through the control of cystathionine $\gamma$ lyase and provide an additional model system to study the dynamics of transsulfuration. In addition, the tight regulation and high expression level of the $N$. crassa cystathionine $\gamma$-lyase gene make it's promoter a potentially valuable tool for studies requiring manipulated control of gene expression.

\section{Results and discussion}

Sequence and characterization of the cystathionine $\gamma$-lyase gene

The nucleotide sequence of the $c y s-16^{+}$gene and flanking $5^{\prime}$ region is presented in Figure 1. The cloning of cys-16 used a cDNA clone, designated as N-EST:Nc3A10, with homology to cystathionine $\gamma$-lyases that had been isolated by the Neurospora Genome Project [18]. The Nc3A10 clone was used to probe a $\lambda-J 1 N$. crassa genomic library by plaque hybridization and the identified clone $\lambda$ used to generate subclones for nucleotide sequencing. The cys-16 $6^{+}$gene, as reported here, was originally isolated prior to the sequencing of the complete $N$. crassa genome and the sequence submitted as GenBank AF401238. Based on cDNA and genomic sequence data the cys $-16^{+}$gene has no introns. A putative transcriptional start CCATCACC is located at -140 upstream of the initiator ATG. Four CYS3 binding sites that were identified in this study (described below) are also annotated in Figure 1 . The cys- $16^{+}$gene encodes a polypeptide of 417 amino acids with a highly conserved pyridoxal 5'phosphate binding site and substrate-cofactor binding pocket [19]. N. crassa cystathionine $\gamma$-lyase shows substantial similarity to fungal (e.g., 77\% identity to Aspergillus nidulans XP_659050 and $60 \%$ identity to Saccharomyces cerevisiae AAC04945)and to human (46\% identity,NP_001893) cystathionine $\gamma$-lyases.

\section{Analysis of cys- $16^{+}$gene expression}

cys- $16^{+}$mRNA size and level were first assayed in $N$. crassa wild-type grown on low and high levels of sulfur (i. e., derepressing and repressing conditions, respectively) . Poly $(\mathrm{A})^{+}$mRNA was isolated, and Northern blots prepared and probed with the cloned $c y s-16^{+}$gene. For the blot shown in Figure 2, mRNA was isolated from cultures grown for $12 \mathrm{~h}$ in low- and high sulfur media (i.e., low- or high-levels of supplemented methionine) inoculated with wild-type conidia. A 1.6-kb message hybridized to the cys$16^{+}$probe and showed a high level of expression under low sulfur (derepressing) growth conditions. In contrast, the cys- $16^{+}$transcript was detectable at only a low level under high sulfur (repressing) growth conditions. The constitutively expressed glutamate dehydrogenase $\left(\mathrm{am}^{+}\right)$ gene was used as a control for ensuring comparability of message levels between the high- and low-sulfur samples. 


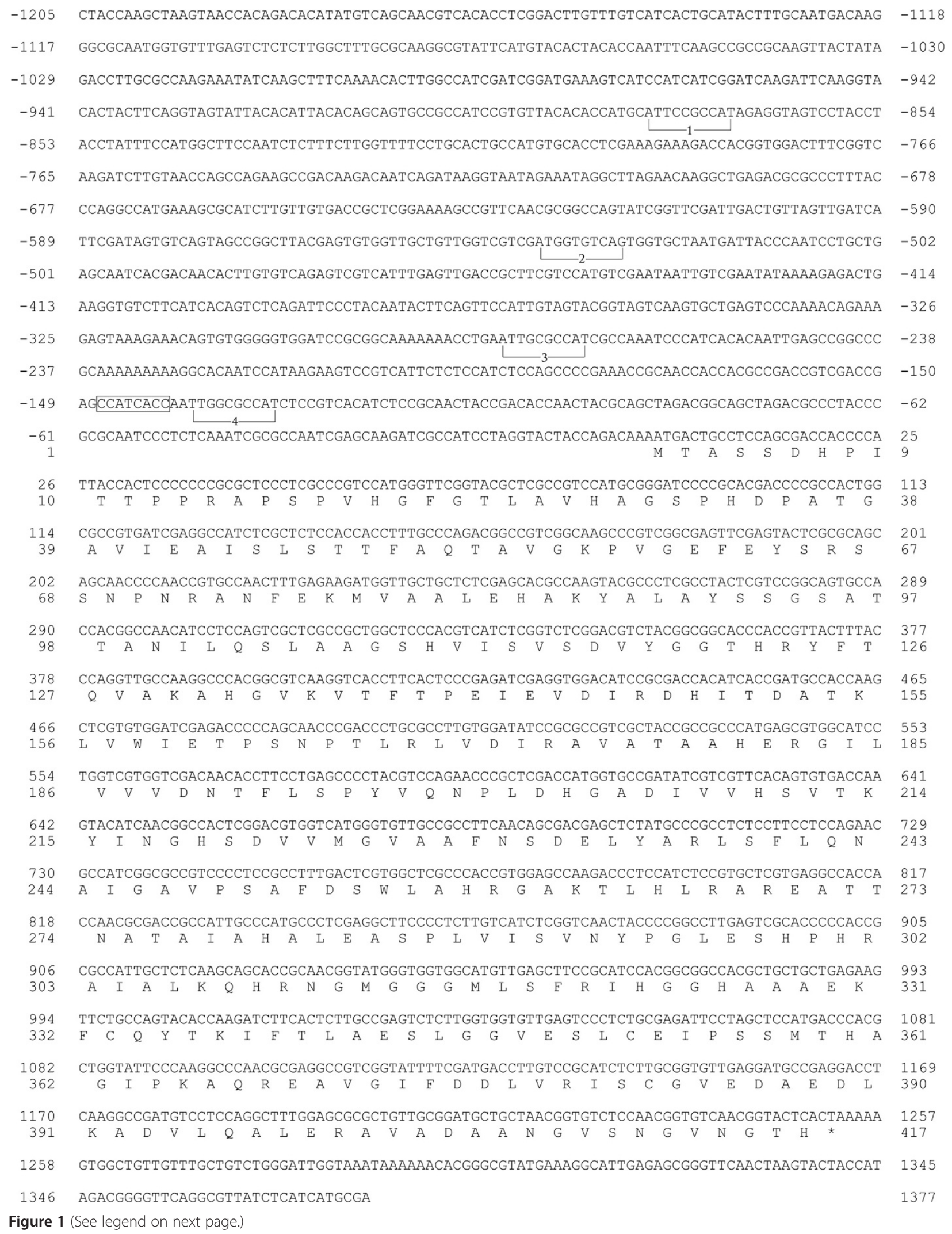


(See figure on previous page.)

Figure 1 Nucleotide and predicted amino acid sequence of the cys-16 $\mathbf{6}^{+}$gene. The sequence is shown from 1205 nucleotides upstream of the translation start codon to 125 nucleotides downstream of the stop codon (indicated by an asterisk). The nucleotides are numbered relative to the initiator ATG codon. CYS3-binding sites within the cys- $16^{+}$promoter are bracketed and numbered 1 through 4 . The sequence corresponding to the $N$. crassa transcriptional initiation site consensus is boxed.

These results suggest that in $N$. crassa, part of the complex response to sulfur limitation (or starvation) involves the derepression of cys-16 $6^{+}$gene expression and consequential increase in cystathionine $\gamma$-lyase activity converting the available pool of cystathionine into cysteine. The dynamics of how transsulfuration is regulated in $N$. crassa appears to be different from other fungal species such as A. nidulans [9] and A. chrysogenum [10], where cystathionine $\gamma$-lyase levels increase under high methionine or are not regulated by methionine level, respectively.

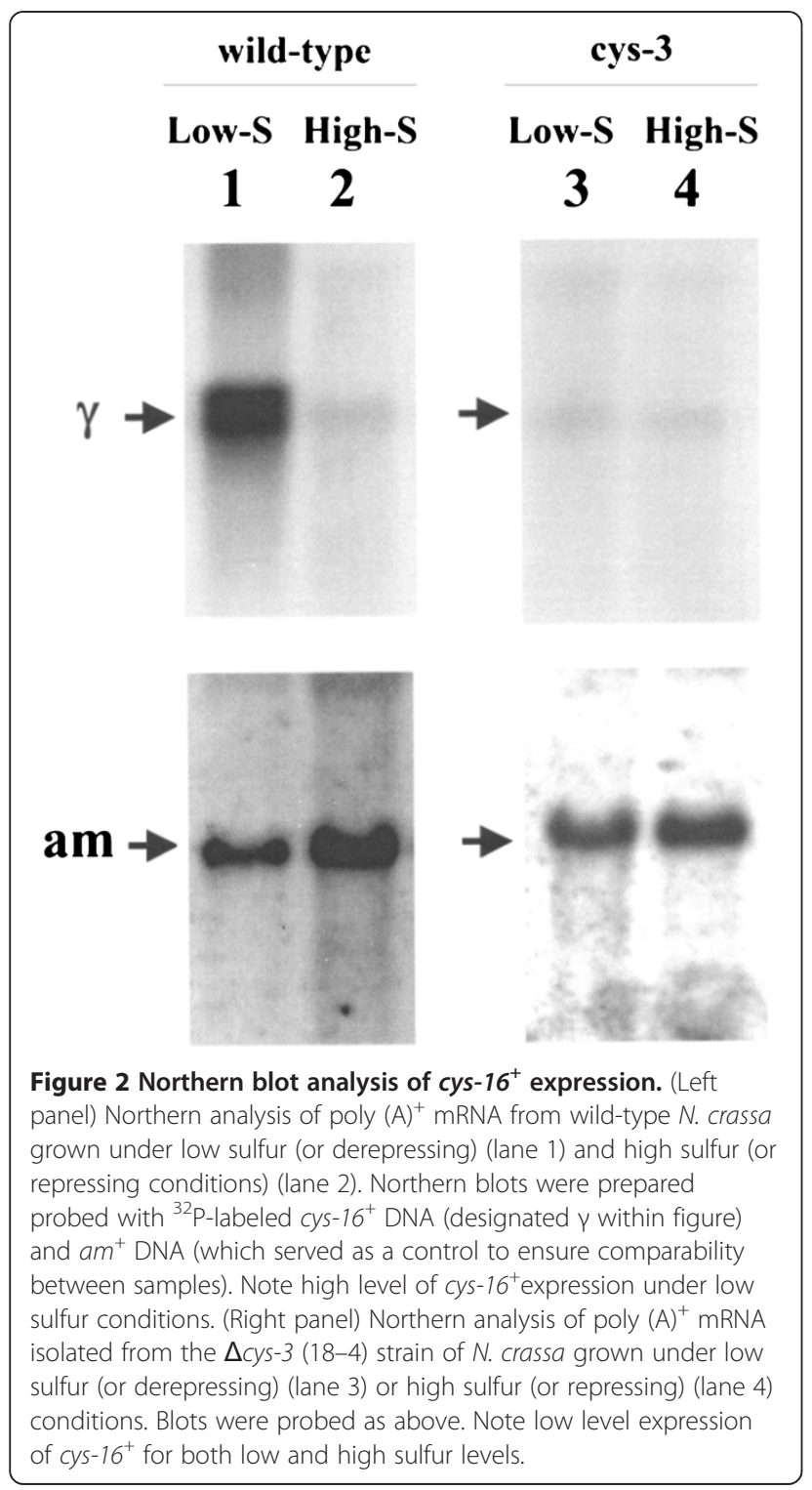

The role of CYS3 in the regulation of cys- $16^{+}$was examined using Northern blot analysis of cys-16 ${ }^{+}$expression in a strain deleted for the cys- $3^{+}$gene $(\Delta c y s-3)$. Northern blots of poly $(\mathrm{A})^{+}$mRNA isolated from $\Delta c y s-3$ grown under low- and high-sulfur conditions were probed with the cys-16 $6^{+}$gene (Figure 2). The level of cys $-16^{+}$transcript detected was equivalently low for both low- and high-sulfur growth conditions; and similar to the repressed level seen in wild-type under high sulfur growth conditions. The $\Delta c y s-3$ mutant completely blocks the derepression of cys- $16^{+}$expression seen under lowsulfur growth conditions in wild-type. In this regard, the cys- $16^{+}$gene is showing the typical response of genes previously confirmed to be part $N$. crassa sulfur control circuit (e.g., arylsulfatase, sulfate permease I and II, choline sulfatase, and others) in that sulfur starvation derepresses transcription in wild-type and mutating the CYS3 regulator abolishes that derepression [11,13].

\section{Gel mobility shift analysis of CYS3 regulator binding}

In order to further confirm the role of CYS3 in the control of cys- $16^{+}$expression, gel mobility shift assays were used to scan the cys-16 $16^{+}$promoter for the presence of CYS3 binding sites. First, the cys- $16^{+}$promoter was divided into 100- to 300-bp segments by restriction endonuclease digestion and analyzed for CYS3 binding with a total of four short segments $(24 \mathrm{bp})$ capable of binding CYS3 being defined (Figure 3). Each short segment contains a core $10 \mathrm{bp}$ sequence that agrees strongly with the consensus binding site for CYS3 (5' ATG GCGC CAT 3') determined by in vitro bindingsite selection [14]. Within the four detected binding sites, a gradation of CYS3 binding affinity in the gel shift assays is present, with CYS3 binding most strongly to site 3 (Figure 3). Note that the core sequence of site 3, $5^{\prime}$ TTG GCGC CAT 3', is the closest match to the consensus CYS3 binding sequence (5' ATG GCGC CAT $\left.3^{\prime}\right)$. Correspondingly, site 1 was the weakest binding site with the poorest match to the consensus CYS3-binding site. To examine the specificity of CYS3 binding to the cys $-16^{+}$promoter sites, a single nucleotide at the sixth position within the 10 base core for each CYS3-binding site was mutated from conserved $G$ to a $T$. The single base mutation eliminated or substantially reduced CYS3 binding at each site. These data also provide a further confirmation of the CYS3 binding-site as determined by in vitro binding-site selection [14]. The definition of 


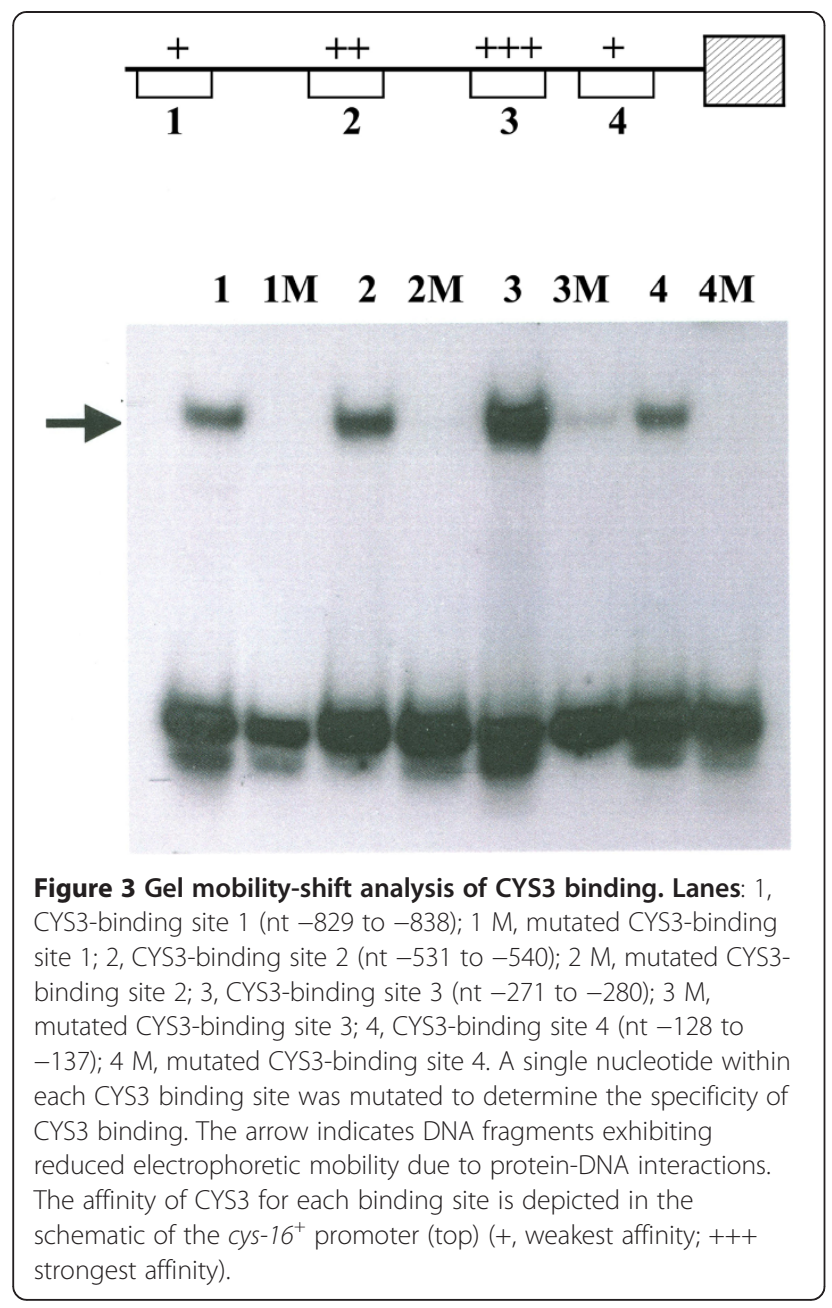

CYS3 binding sites to the $c y s-16^{+}$promoter also provides necessary information for using the cys- $16^{+}$promoter as gene expression tool in $N$. crassa.

\section{Conclusions}

The cystationine $\gamma$-lyase gene, cys- $16^{+}$, is tightly regulated and is derepressed upon growth of $N$. crassa under conditions of sulfur limitation. Taken collectively, the in vivo expression experiments using a $\Delta c y s-3$ mutant and the in vitro CYS3 binding data strongly supports the CYS3 regulation of cys- $16^{+}$expression. These experiments provide a starting point for examining the dynamics of transsulfuration control in a model eukaryotic system. In addition, the cys-16 gene contains a potentially useful regulatable promoter for gene expression studies.

\section{Methods}

\section{Strains, plasmids and culture conditions}

74OR23-1a was used as the wild-type (WT) for these studies. $\Delta c y s-3$ (18-4) was constructed and described in a prior study [20]. The $\lambda-J 1 N$. crassa genomic library and N-EST:Nc3A10 clone were obtained from the Fungal Genetics Stock Center (Kansas City, KS). Vogel minimal medium [21], with supplements as required, was used. N. crassa cultures were grown at $25^{\circ}$ C. Sulfur repression and derepression experiments were done by growth of mycelia on Vogel-minus-sulfur medium plus high sulfur (5.0 $\mathrm{mM}$ methionine) and low-sulfur medium (0.25 $\mathrm{mM}$ methionine) medium, respectively.

\section{Gene cloning and sequencing}

The $N$. crassa cDNA clone N-EST:Nc3A10 was used to produce a ${ }^{32} \mathrm{P}$-labeled probe and carry out plaque hybridization as described [22] to a $N$. crassa $\lambda$-J1 genomic library and identify hybridizing clones. A segment containing the cys-16 gene derived from an isolate designated $\lambda 412$ was subsequently subcloned into pSPORT and subjected to automated sequencing (Cleveland Genomics; Cleveland, $\mathrm{OH}$ ) by primer walking. The genomic sequence was submitted to GenBank as AF401238. Following the cloning of the gene, the gene symbol cys- $16^{+}$was assigned by A. Radford (University of Leeds) and included in The Neurospora crassa eCompendium [23]. The gene has also since been given the locus designation NCU09230 in the Broad Institute Neurospora crassa database [24].

\section{Northern analysis}

Poly $(\mathrm{A})^{+}$mRNA was isolated by phenol extraction and subsequent oligo (dT)-cellulose chromatography as described previously [20]. Briefly, mycelial samples were harvested by filtration, frozen in liquid nitrogen, and homogenized in a 1:1 mixture of phenol-chloroformisoamyl alcohol (49:49:2) and extraction buffer (1\% sarkosyl, $100 \mathrm{mM}$ sodium acetate, $1 \mathrm{mM}$ EDTA [pH 5.0]). After phenol-chloroform extractions, precipitation, and sodium acetate washes, and the poly $(\mathrm{A})^{+}$mRNA was isolated by oligo (dT)-cellulose chromatography. ${ }^{32} \mathrm{P}$-labeled probes were prepared by oligolabeling of DNA fragments [25]. Northern blots were hybridized and washed as outlined elsewhere [20].

\section{Gel mobility shifts}

Purified promoter fragments derived from restriction endonuclease digestion were used in an initial scan for CYS3 binding. Consequently, oligonucleotides representing the binding sites to be tested were synthesized (Applied Biosystems 391EP synthesizer), labeled by T4 polynucleotide kinase with $\left[\gamma^{-}{ }^{32} \mathrm{P}\right] \mathrm{ATP}$, annealed and gel purified as described $[14,26]$. The following oligonucleotides (and complementary strands) representing the four putative CYS3 binding sites on the $c y s-16^{+}$promoter were prepared: Site 1 [5' ACCATGCATTCCGCCATAGAGGTA 3'], Site 2 [5' GTCGTCGATGGTGTCAGTGGTGCT 3'], Site 3 [5' AACCTGAATTGCGCCATAGCCAAA 3'], and Site 4 
[5' TCACCAATTGGCGCCATCTCCGTC 3']. The synthesized mutated versions had a $\mathrm{G}$ to $\mathrm{T}$ substitution at the sixth position of the $10 \mathrm{bp}$ core of the CYS3 consensus binding site: Site $1 \mathrm{M}$ [5' ACCATGCATTCCTCCATAGAGGTA 3'], Site $2 \mathrm{M}$ [5' GTCGTCGATGGGTTCAGTGGTGCT 3'], Site 3 M [5' AACCTGAATTGCT CCATCGCCAAA $\left.3^{\prime}\right]$, and Site 4 M [5' TCACCAATTGGCTCCATCTCCGTC 3']. DNA-binding assays were carried out as we have described previously using Eschericia coli produced CYS3 protein $[14,20]$. Specificity of binding was ensured by control experiments using competition by addition of excess unlabeled DNA. Four percent PAGE gels with a $50 \mathrm{mM}$ Tris- $80 \mathrm{mM}$ glycine2 mM EDTA (pH 8.5) running buffer were electrophoresed at $20 \mathrm{~mA}$ with the temperature maintained at $4^{\circ} \mathrm{C}$. Quantitation of gel shift assays was performed by using a Molecular Dynamics Phosphorimager.

\section{Availability of supporting data}

The sequence data supporting the results of this article is available in the GenBank repository [AF401238, http://www.ncbi.nlm.nih.gov/nuccore/AF401238].

\section{Competing interests}

The authors declare that they have no competing interests.

\section{Authors' contributions}

BR carried out the majority of experiments. JP and BR designed the experiments and prepared the manuscript. Both authors read and approved the final manuscript.

\section{Acknowledgements}

The work was supported by a Medical Innovations Grant from the Wright State University Boonshoft School of Medicine.

Received: 16 January 2012 Accepted: 5 June 2012

Published: 2 July 2012

\section{References}

1. Chiku T, Padovani D, Zhu W, Singh S, Vitvitsky V, Banerjee R: $\mathrm{H}_{2} \mathrm{~S}$ biogenesis by human cystathionine $\gamma$-lyase leads to the novel sulfur metabolites lanthionine and homolanthionine and is responsive to the grade of hyperhomocysteinemia. J Biol Chem 2009, 284:11601-11612.

2. Singh $S$, Padovani D, Leslie RA, Chiku T, Banerjee R: Relative contributions of cystathionine $\beta$-synthase and $\gamma$-cystathionase to $\mathrm{H}_{2} \mathrm{~S}$ biogenesis via alternative trans-sulfuration reactions. J Biol Chem 2009, 284:22547-22466.

3. Yang G, Wu L, Yang W, Qi J, Cao K, Meng Q, Mustafa AK, Mu W, Zhang S, Snyder $S$, Wang $R: \mathrm{H}_{2} \mathrm{~S}$ as a physiologic vasorelaxant: hypertension in mice with deletion of cystathionine $\gamma$-lyase. Science 2008, 322:587-590

4. Flavin M: Trans-sulfuration reactions: an introduction. Methods Enzymol 1971, 17B:416-417

5. Steegborn C, Clausen T, Sondermann P, Jacob U, Worbs M, Marinkovic S, Huber R, Wahl MC: Kinetics and inhibition of recombinant cystathionine gamma-lyase Toward the rational control of transsulfuration. J Biol Chem 1999, 274:12675-12684.

6. Flavin M, Segal A: Purification and properties of the cystathionine Y-cleavage enzyme of Neurospora. J Biol Chem 1964, 239:2220-2227.

7. Flavin M, Slaughter C: The derepression and function of enzymes of reverse trans-sulfuration in Neurospora. Biochim Biophys Acta 1967, 132:406-411.

8. Burton EG, Metzenberg RL: Novel mutation causing derepression of several enzymes of sulfur metabolism in Neurospora crassa. J Bacterio 1972, 109:140-151.
9. Sienko M, Natorff R, Owczarek S, Olewiecki I, Paszewski A: Aspergillus nidulans genes encoding reverse transsulfuration enzymes belong to homocysteine regulon. Curr Genet 2009, 55:561-570.

10. Marcos At, Kosalkova K, Cardoza RE, Fierro F, Gutierrez S, Martin JF: Characterization of the reverse transsulfuration gene mecB of Acremonium chrysogenum, which encodes a functional cystathionine- $\gamma$ lyase. Mol Gen Genet 2001, 264:746-754.

11. Paietta JV: Sulfur, phosphorus and iron metabolism. In Cellular and molecular biology of filamentous fungi. Edited by Borkovich KA, Ebbole DJ. Washington: American Society for Microbiology; 2010:359-375.

12. Borkovich KA, Alex LA, Yarden O, Freitag M, Turner GE, Read ND, Seiler $S$, Bell-Pederson D, Paietta JV, Pesofsky N, Plamann M, Goodrich-Tanrikulu M, Schulte U, Mannhaupt G, Nargang FE, Radford A, Selitrennikoff C, Galagan JE, Dunlap JC, Loros J, Catheside D, Inoue H, Aramayo R, Polymenis M, Selker EU, Sachs MS, Marzluf GA, Paulsen I, Davis R, Ebbole DJ, et al: Lessons from the genome sequence of Neurospora crassa: tracing the path from genomic blueprint to multicellular organism. Microbiol Mol Rev 2004, 68:1-108.

13. Paietta JV: Regulation of sulfur metabolism in mycelial fungi. In The mycota. III. Biochemistry and molecular biology. second edth edition. Edited by Brambl R, Marzluf GA. Berlin: Sprinter-Verlag; 2004:369-383.

14. Paietta JV: DNA-binding specificity of the CYS3 transcription factor of Neurospora crassa defined by binding-site selection. Fungal Genet Biol 2008, 45:1166-1171.

15. Kasuga T, Townsend JP, Tian C, Gilbert LB, Mannhaupt G, Taylor JW, Glass $\mathrm{NL}$ : Long-oligomer microarray profiling in Neurospora crassa reveals the transcriptional program underlying biochemical and physiological events of conidial germination. Nucleic Acids Res 2005, 33:6469-6485.

16. Tian C, Li J, Glass NL: Exploring the bZIP transcription factor regulatory network in Neurospora crassa. Microbiology 2011, 157:747-759.

17. Videira A, Kasuga T, Tian C, Lemos C, Castro A, Glass NL: Transcriptional analysis of the response of Neurospora crassa to phytosphingosine reveals links to mitochondrial function. Microbiology 2009, 155:3134-3141.

18. Nelson MA, Kang S, Braun EL, Crawford ME, Dolan PL, Leonard PM, Mitchell J, Armijo AM, Bean L, Blueyes E, Cushing T, Errett A, Fleharty M, Gorman M, Judson K, Miller R, Ortega J, Pavlova I, Perea J, Todisco S, Trujillo R, Valentine J, Wells A, Werner-Washburne M, Yazzie S, Natvig DO: Expressed sequences from conidial, mycelial, and sexual stages of Neurospora. Fungal Gene Biol 1997, 21:348-363.

19. Alexander FW, Sandmeier PK, Christen P: Evolutionary relationships among pyridoxal-5'-phosphate-dependent enzymes. Eur J Biochem 1994, 219:953-960.

20. Paietta JV: Production of the CYS3 regulator, a bZIP DNA-binding protein, is sufficient to induce sulfur gene expression in Neurospora crassa. Mol Cell Biol 1992, 12:1568-1577.

21. Davis RH, DeSerres EF: Genetic and microbiological research techniques for Neurospora crassa. Methods Enzymol 1970, 17A:79-143.

22. Paietta JV: Molecular cloning and regulatory analysis of the arylsulfatase structural gene of Neurospora crassa. Mol Cell Biol 1989, 9:3630-3637.

23. The Neurospora crassa e-Compendium. http://bmbpcu36.leeds.ac.uk/ gen6ar/newgenelist/genes/index.html.

24. Broad Institute Neurospora crassa database. http://www.broadinstitute.org/ annotation/genome/neurospora/MultiHome.html.

25. Feinberg AP, Vogelstein B: A technique for radiolabelling DNA restriction endonuclease fragments to high specific activity. Anal Biochem 1983, 132:6-13.

26. Kumar A, Paietta JV: An additional role for the F-box motif: gene regulation within the Neurospora crassa gene control network. Proc Nat Acad Sci USA 1998, 95:2417-2422.

doi:10.1186/1756-0500-5-339

Cite this article as: Reveal and Paietta: Analysis of the sulfur-regulated control of the cystathionine $y$-lyase gene of Neurospora crassa. BMC Research Notes 2012 5:339. 